\title{
Color Image Enhancement by an Integral Mask-filtering Approach Employing Nonlinear Transfer Function
}

\author{
Ching-Chung Yang \\ Department of Multimedia Design, Tatung Institute of Commerce and Technology, 253 Mi-Tuo Road, Chiayi City, Chinese Taipei. \\ Email: yang10.cc@msa.hinet.net
}

Received 2013

\begin{abstract}
We demonstrate a brand-new method to sharpen a color image by using an integral mask-filtering technique. The derivatives between the target pixel and its neighbors are transferred by the cubic root function instead of the traditional linear one. The obtained final image has clearer fine characteristics along with much less overshooting.
\end{abstract}

Keywords: Image Enhancement; Mask-filtering; Transfer Function; Overshooting

\section{Introduction}

The mask-filtering approach is widely used to sharpen images by the implementation of the Laplacian operator $[1,2]$. For a $3 \times 3$ mask of [-1 -1 -1; $-18-1 ;-1-1-1]$, when used to sharpen an image $f(m, n)$, the second derivative could be expressed as the following:

$$
\begin{aligned}
\nabla^{2} f(m, n)= & 8 f(m, n)-[f(m-1, n-1) \\
& +f(m-1, n)+f(m-1, n+1)+f(m, n-1) \\
& +f(m, n+1)+f(m+1, n-1)+f(m+1, n) \\
& +f(m+1, n+1)]
\end{aligned}
$$

Eq. (1) could be summed up as the following:

$$
\begin{gathered}
\nabla^{2} f(m, n)=A \times \sum_{i=-1}^{1} \sum_{j=-1}^{1}[f(m, n)-f(m+i, n+j)] \\
\text { where }(i, j) \neq(0,0)
\end{gathered}
$$

There are totally eight derivatives calculated in Eq. (2). This summation is multiplied by a coefficient A. It is then imposed on the original image $f(m, n)$ to get an enhanced new one $\mathrm{g}(\mathrm{m}, \mathrm{n})$ as the following:

$$
g(m, n)=f(m, n)+\nabla^{2} f(m, n)
$$

Eq. (3) is commonly used to obtain sharpened pictures with satisfactory results in general conditions. While this mask-filtering technique has some annoying drawbacks. The major problem is that it sums up all the derivatives between the target pixel and the surrounding neighbors in a time. Although this summation could noticeably change the target's own grey-level. Such change at times appears over-exaggerated. In consequence, there is often some unwanted overshooting happened on the image objects after this processing.
The conceptual masking filter is qualitatively shown in Figure 1 with a $4 \times 4$ matrix. The target pixel is surrounded by eight neighbors in different directions. When using this filter with the traditional transferring, it seems not avoidable to derive a much larger output via big inputs. Hence, the nonlinear calculation is substituted here for the linear one in Eq. (2) to reduce the overshooting. In this work, the cubic root function is selected as the substitution. The big inputs are expected to gradually saturate after the transferring, while the smaller ones could derive larger outputs. Hopefully, the processed image would be with less overshooting as well as clearer fine characteristics.

\section{Algorithm}

The cubic root function is chosen here for that its curve increases rapidly in lower values while saturates gradually in higher ones. Its curve is shown in Figure 2 in comparison with the linear transferring.

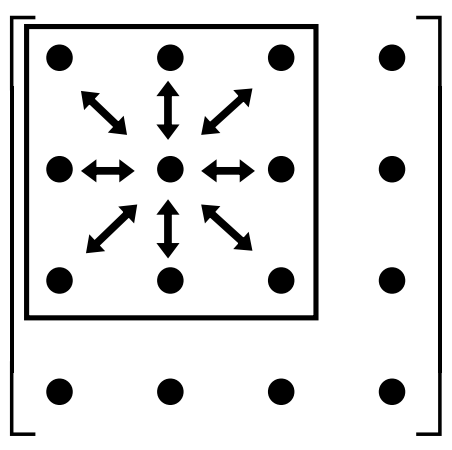

Figure 1. Conceptual diagram of the masking filter. The target pixel changes its value by exploiting its eight neighbors oriented in different directions. 


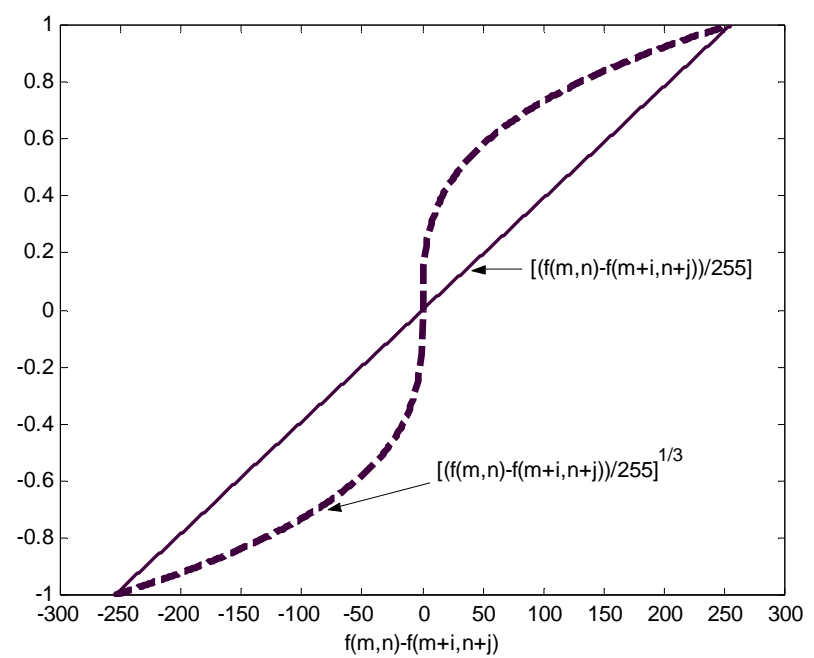

Figure 2. Comparison of two different transfer functions. The solid line is for the transfer curve in Eq. (2), while the dash line is for that in Eq. (4).

When choosing this new transfer function, we modify the target pixel increment from Eq. (2) to be the following:

$$
\begin{gathered}
\Delta f(m, n)=B \times \sum_{i=-1}^{1} \sum_{j=-1}^{1}\left[\frac{(f(m, n)-f(m+i, n+j))}{255}\right]^{1 / 3} \\
\text { where } \quad(i, j) \neq(0,0)
\end{gathered}
$$

Meanwhile, this increment should multiply together a coefficient B to adapt to different processed picture. And Eq. (3) is then changed to the following:

$$
g(m, n)=f(m, n)+\Delta f(m, n)
$$

The term $f(m, n)-f(m+i, n+j)$ in Eq. (4) actually represents the high frequency component as we mentioned before [3-9]. But the low frequency component of the image, the term $f(m, n)+f(m+i, n+j)$, is not yet introduced into the masking. That means the brightness distribution of the whole image still remains unadjusted after the above processing.

Hence, we introduce a second mask to take into account this consideration as the following:

$$
\begin{aligned}
\bar{f}(m, n)= & C \times \sum_{i=-1}^{1} \sum_{j=-1}^{1}\left[\frac{f(m, n)+f(m+i, n+j)}{2 \times 255}\right]^{11 / 10} \\
& \text { where }(i, j) \neq(0,0)
\end{aligned}
$$

The term $(f(m, n)+f(m+i, n+j)) / 2$ in Eq. (6) represents the low frequency component of the image, and coefficient $C$ would affect its amplitude. The power 11/10 here could be replaced by larger value in case that the picture is non-uniformly illuminated. Therefore, the illumination adjusting of the full image is going to be accomplished by using this second mask. The result image $\mathrm{g}(\mathrm{m}, \mathrm{n})$ is finally obtained by an integral mask-filtering as the following:

$$
g(m, n)=2 \times f(m, n)-\bar{f}(m, n)+\Delta f(m, n)
$$

Eq. (7) means that a much better enhanced image could be acquired by subtracting the primary low frequency components alongside of imposing the crucial high frequency ones.

The coefficients A, B and C in the above equations are experimentally determined here to obtain better output results.

\section{Experiment}

We use Matlab 7.0 to deal with this experiment. The HSV color system is selected here owing to its convenient acquisition from the Matlab tool box. A color image in this system is considered to comprising three components including hue, saturation, and value. The value component represents the brightness of a colorful image. It is similar to the gray-level magnitude of a colorless picture. Then we can apply the mentioned algorithm onto the colored images scope.

The input image shown in Figure 3(a) has a $256 \times 256$ dimension., We use the traditional mask-filtering approach to get Figure 3(b), which is derived by using Eq. (2) and Eq. (3) with coefficient A = 1/3. There we could see many unwanted overshooting happened on the object edges in the picture. Figure 3(c) is the processed result by Eq. (4) and Eq. (5) with coefficient B = 12. There the fine characteristics are better enhanced with much less overshooting. And Figure 3(d) is the final result by using Eq. (6) and Eq. (7) with coefficient $\mathrm{C}=35$. The image sharpening is now further reinforced by adjusting the image illumination.

\section{Discussions and Conclusions}

By comparing Figure 3(b)-(d), some merits are found upon the usage of our proposed method. The most important is that this integral masking is capable of revealing more details along with less overshooting.

In Figure 3(c), there is much less overshooting happened on the tree branches and leaves. While its grass and buildings are clearer than those in Figure 3(b). This could be deduced that the nonlinear transferring rather than the linear one is more promising when using the mask-filtering approach. And by adjusting the illumination, Figure 3(d) shows that the attic roof is better distinguished. This is because that the local visibility has been improved by reducing the low frequency components.

Although the increments in Eq. (2), (4) and (6) are scalars, there are directional messages hidden inside them. For that they are summations of derivatives along different directions. But these hidden messages tend to distort once their values easily got saturated, just like 


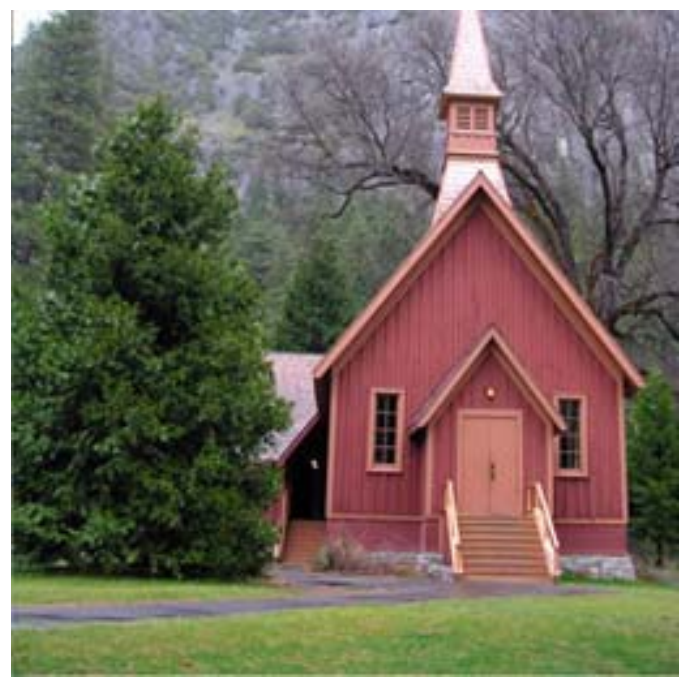

(a)

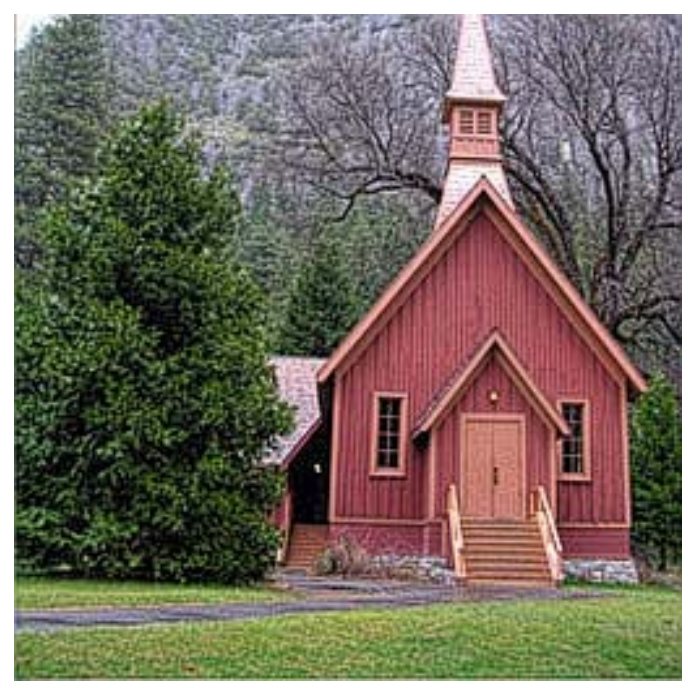

(c)

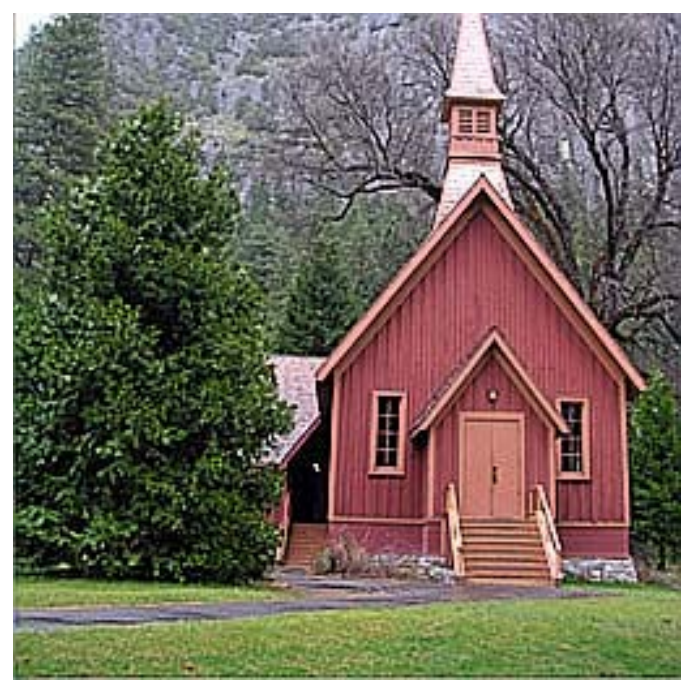

(b)

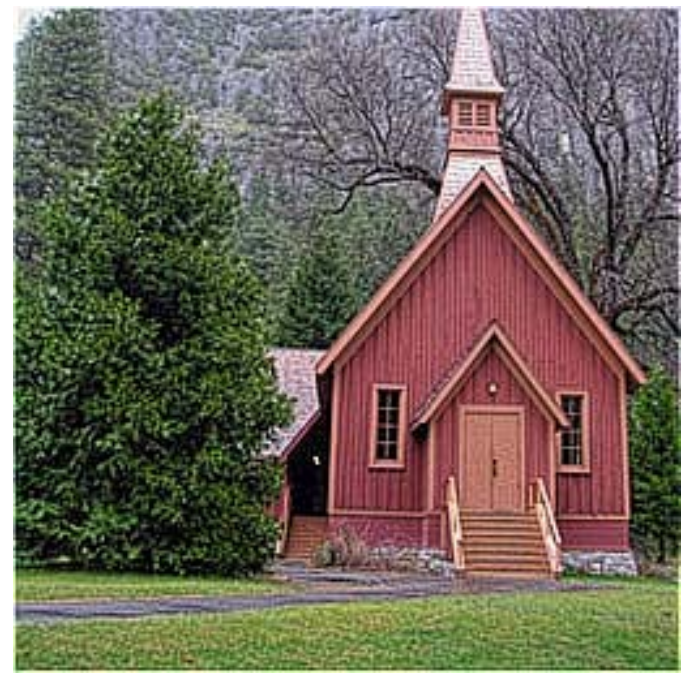

(d)

Figure 3. (a) The original image. (b) The processed image by using the traditional Eq. (3). (c) The processed image by using Eq. (5). (d) The processed image by using Eq. (7).

those found in the traditional linear transferring. While by using the proposed method, such problem could be distinctly suppressed. The demonstrated technique is useful for various applications, such as medical image enhancement, remote sensing, microscopic image sharpening, etc.

\section{REFERENCES}

[1] W. K. Pratt, "Digital Image Processing," Wiley, New York, 2001, pp. 278-284. doi:10.1002/0471221325

[2] R. C. Gonzalez and R. E. Woods, "Digital Image Processing,” Prentice Hall, New Jersey, 2001, pp. 125-132.

[3] C. C. Yang, "Improving the Sharpness of An Image with Non-Uniform Illumination”, Optics and Laser Technology, Vol. 37, 2005, pp. 235-238. doi:10.1016/j.optlastec.2004.03.015

[4] C. C. Yang, "Image Enhancement by Modified Contrast-Stretching Manipulation”, Optics and Laser Technology, Vol. 38, No. 2006, pp. 196-201. doi:10.1016/j.optlastec.2004.11.009

[5] C. C. Yang, "Image Enhancement by Adjusting the ConTrast of Spatial Frequencies,” Optik, Vol. 119, 2008, pp. 143-146. doi:10.1016/j.ijleo.2006.07.011

[6] C. C. Yang, "Image Enhancement by the Modified High-Pass Filtering Approach”, Optik, Vol.120, 2009, pp. 886-889. doi:10.1016/j.ijleo.2008.03.016

[7] C. C. Yang, "A Modification for the Mask-Filtering Approach by Superposing Anisotropic Derivatives in An Image,” Optik, Vol.122 , 2011, pp. 1684-1687. doi:10.1016/j.ijleo.2010.10.026

[8] C. C. Yang, "Color image enhancement by a modified 
mask-filteringapproach,” Optik, Vol. 123, 2012, pp. 1765-1767. doi:10.1016/j.ijleo.2011.11.057

[9] C. C. Yang, "Improving the Overshooting of A Sharp- ened Image by Employing Nonlinear Transfer Functions in the Mask-Filtering Approach,” Optik, in press. 